\title{
A TECHNIQUE FOR THE STUDY OF THE INTERNAL STRUCTURE OF CALCIFIED TISSUES
}

\author{
DONALD E. KNAPP, JAMES K. AVERY, AND EMMETT R. COSTICH \\ Department of Oral Histology and Embryology, University of Michigan, School of Dentistry, \\ Ann Arbor, Mich.
}

$T^{H E}$ investigations of Williams and Irvine $e^{1}$ showed that extraction of organic material from bone with ethylenediamine (ED) was superior to ashing with $\mathrm{KOH}$-glycol. Since the anorganic skeleton prepared in the former manner appeared to be intact, the method was utilized by them in studies of trabecular stress patterns. They also noted that less alteration of crystalline structure resulted from preparation of the anorganic bone from ED than from glycolashing methods. Peckham and Losee ${ }^{2}$ eompared the efficiency of $\mathrm{ED}$ and $\mathrm{KOH}-$ glycol extractions of dentin and found that there was a negligible loss of mineral content with the former method in contrast to an appreciable amount with the latter. This is believed to be due to the relatively low temperature $\left(118^{\circ}\right.$ C.) used in removing the organic fractions with $\mathrm{ED}$ in a modified Soxhlet extractor. Because ethylenediamine is readily soluble in water, it can easily be washed out of the anorganic matrix after extraction. Losee ${ }^{3}$ utilized ED extraction to study the mieroseopie pathways in enamel and demonstrated the presence of micro-lamellae in human enamel. It was the aim of the present investigation to utilize this improved method of extraction of organic components of calcified tissues in developing a method of studying the internal structure of these tissues.

\section{METHODS AND MATERIAIS}

Fresh samples of bone and teeth were placed immediately in fritted disk, Pyrex extraction thimbles. The thimbles then were placed in modified Soxhlet extractors (Lose ${ }^{4}$ ) and the samples were extracted at $118^{\circ} \mathrm{C}$. with a solution of ethylenediamine, consisting of 80 parts of $\mathrm{ED}$ and 20 parts of distilled water. One hundred milliliters of the solution of ethylenediamine was found sufficient to extract the organic material from samples of fresh bone or teeth up to 5 grams in weight. The extraction was carried out in a hood because of the toxicity of the boiling ethylenediamine. The period of extraction was dependent upon the size of the sample. An average of 48 to 72 hours was found to be necessary for a 5-gram sample. The extracted tissues then were washed for 30 minutes in a constantly ehanging bath of distilled water previously shaken with anorganie bone powder and filtered. After dehydration in a series of ascending alcohols

Read at the 36th Annual Meeting of the International Association for Dental Research, Detroit, Michigan, March, 1958.

Received for publication April 2, 1958. 
and subsequent removal of the alcohol with ethyl ether, one series of anorganie bone was placed in unsaturated polyester resin liquid (molecular weight approximately 2,000) containing a particulate dyestuff* and transferred to a vacuum chamber. The vacuum was maintained for 30 minutes at a level just below the point of ebullition of the liquid plastic. Then the vacuum was reduced, catalyst was added, and the sample cured. The dye was retained on the walls of the vascular channels while clear plastic filtered through the haversian eanals and filled the lacunae, canaliculi, and any other small spaces present. The specimens then were decalcified with $0.1 \mathrm{~N} \mathrm{HCl}$. After dehydration, a second impregnation with unsaturated polyester resin resulted in an optically clear specimen except for the vascular channels which were effectively outlined throughout the block with the dyestuff of the initial infiltration.

A modification of this double-embedding technic for bone was accomplished by only partially infiltrating the secondary plastic mass into the specimen. In this manner an interface was gained between the cleared portion of the block and that portion containing plastic in only the spaces previously containing organic matter. Thus, at any desired level in the block, the microstructure of the bone could be revealed. (It should be noted that the curing temperature and the amount of catalyst used in both impregnations must be constant to prevent clouding of the otherwise clear plastic.)

In the third series, the extracted teeth were washed, dehydrated in alcohol, and placed in ethyl ether. The specimens then were quickly transferred into a 60-40 mixture of methyl and butyl methacrylate monomers. Since these monomers have small molecular volumes (in the range of 160 cubic $\AA$ ), impregnation into very small spaces can be achieved. The volume of the monomer was at least 100 times that of the specimen. To ensure complete impregnation, the tissue was allowed to remain in the monomer for 72 hours. The catalyst $\dagger$ then was added and the plastic eured for 72 hours at $60^{\circ} \mathrm{C}$. The sample was decalcified, washed with distilled water, and infiltrated with gelatin. The gelatin was introduced by placing the specimen in a freshly prepared 10 per cent solution for 1 week at $60^{\circ} \mathrm{C}$., during which time the aqueous solution was allowed to dehydrate to one half its original volume. The gelatin was gelled in the refrigerator and further hardened by immersion in 10 per cent formalin. These specimens were sectioned at approximately 5-10 $\mu$ on the freezing microtome. The resulting plastic-gelatin matrix was sufficiently contrasting to permit microscopie study.

\section{OBSERVATIONS AND DISCUSSION}

Ethylenediamine-treated cortical bone appears white, porous, and ashlike as seen in Fig. 1. After impregnation with a plastic containing a dyestuff, decalcification and re-impregnation with a clear plastic, the vaseular channels become apparent (Fig. 2). In Fig. 3 a higher magnification of Fig. 2 is seen.

*Ward's BPM Opaque Color, Ward's Natural Science Establishment, Inc, Rochester, N. Y.

$\dagger$ Luperco C D B, Lucidol Division of the Novadel Agene Corp., Buffalo, N. Y. 

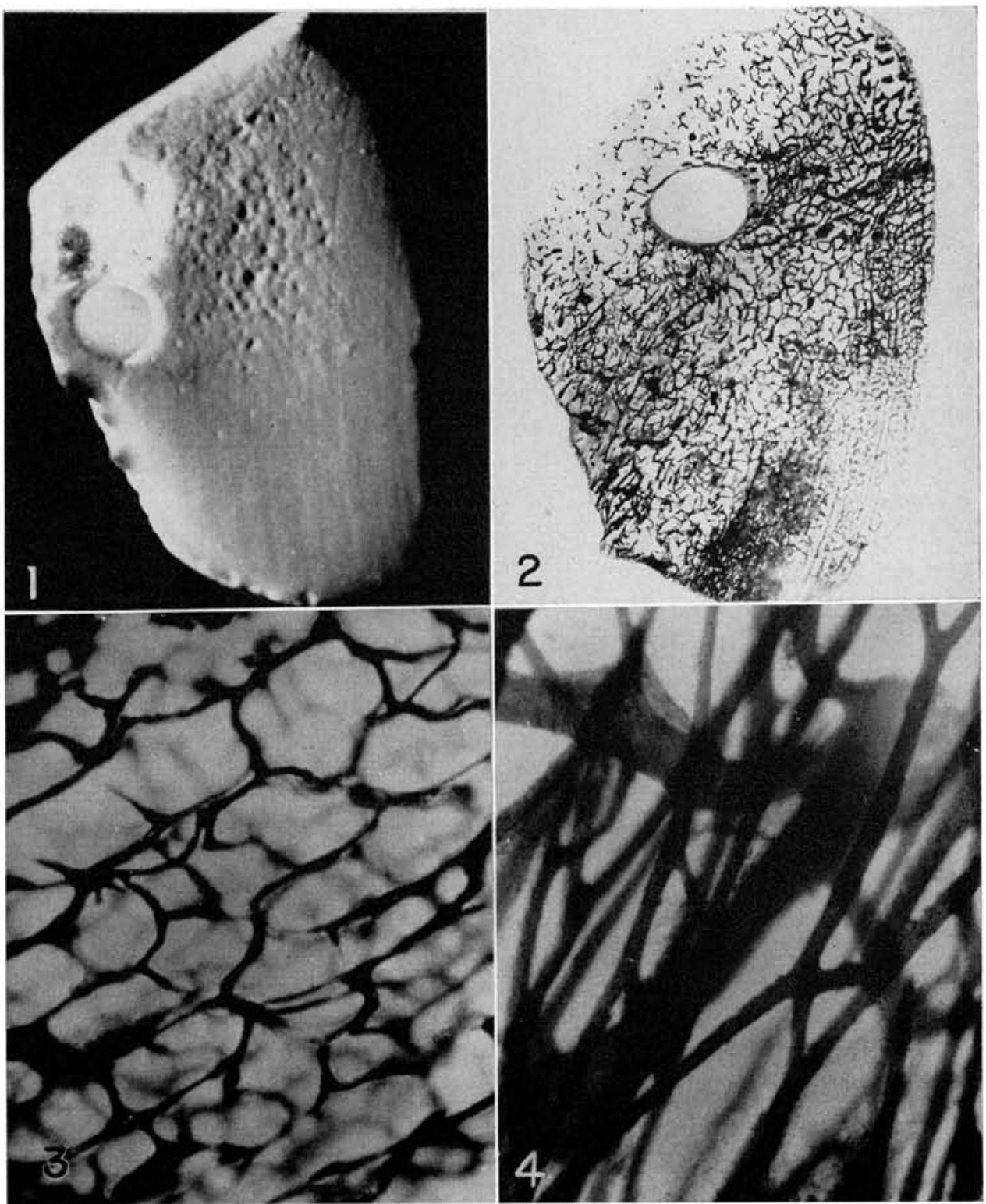

Fig. 1.-Anorganic bone. Bovine cortical bone was cut in sections and treated with ethylenediamine to remove all organic material.

Fig. 2.-An adjacent section of the bone, shown in Fig. 1, impregnated with plastic bearing a particulate dyestuff, then decalcified and reimpregnated with clear plastic. During the initial impregnation, the walls of the haversian canals acted as a filter to hold back the dye particles while the clear plastic penetrated into the smaller spaces previously occupied by organic matter in the specimen. Subsequent demineralization and reimpregnation with clear plastic cleared the specimen. The numerous vascular channels and their branches can be seen.

Fig. 3.-A higher magnification of Fig. 2 reveals the distribution and configuration of some of the vascular channels outlined with dye in the otherwise cleared specimens. The canals are viewed in a plane perpendicular to the long axis of the bone.

Fig. 4.-Another block of cortical bone treated as before but seen here in the long axis of the bone. This specimen is $300 \mu \mu$ thick and clearly reveals the superficial as well as the deeper channels in the cleared plastic specimen. The smallest channels measure $25 \mu \mu$ in diameter. 


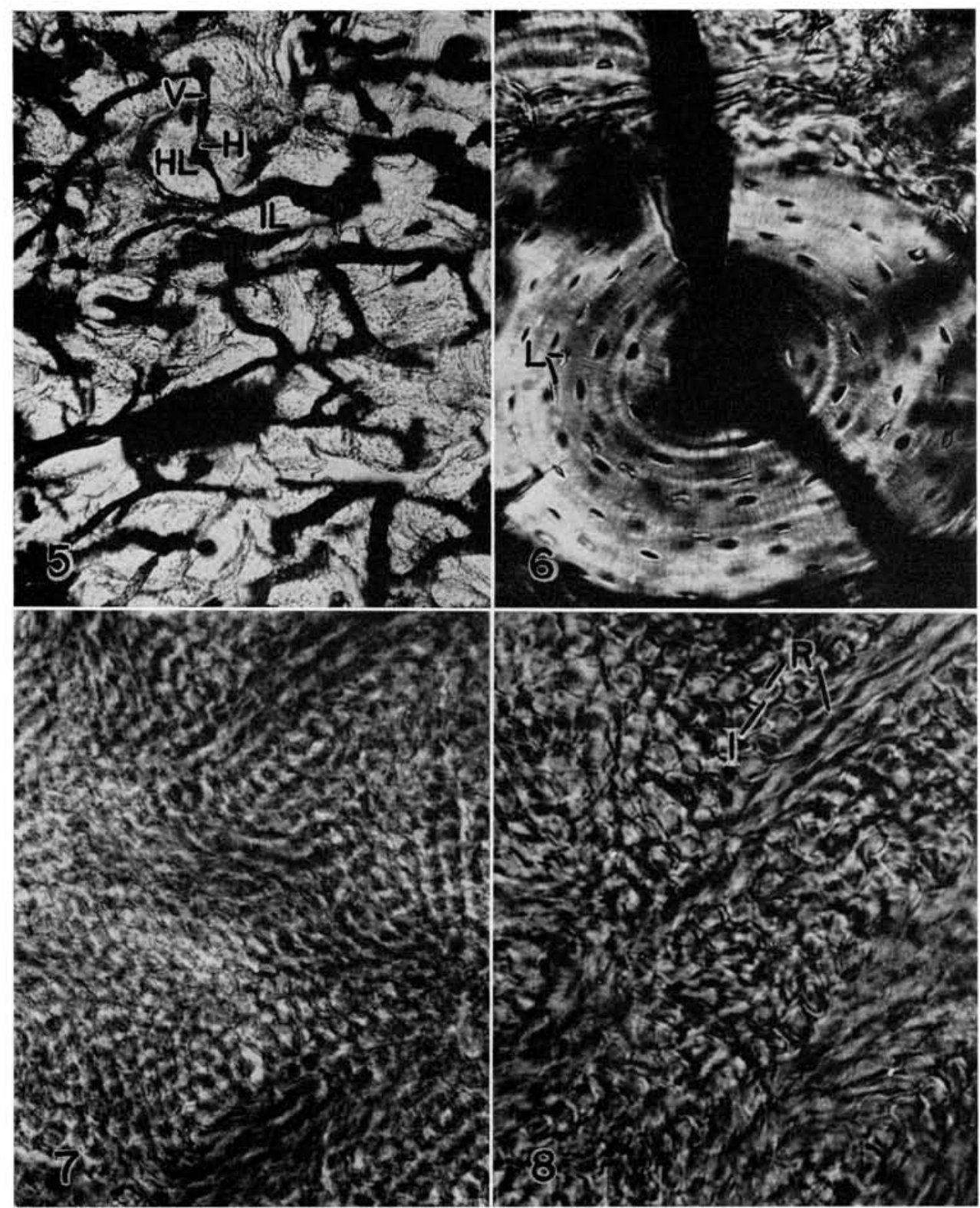

Fig. 5.-After partial secondary plastic impregnation and using oblique illumination, the microscopic structure of haversian systems can be observed as well as the related vascular channels, Volkmann's canals $(V)$, haversian canals $(H)$, interstitial lamellae $(I L)$, and haversian lamellae $(H L)$

Fig. 6.-A higher magnification of Fig. 4 reveals detailed structure of an haversian system. Note the haversian lamellae, lacunae, and canaliculi and two Volkmann's canals joining the haversian canal. Lacunae $(L)$.

Fig. 7.-This is a phase contrast photomicrograph of a plastic and gelatin model of mature enamel. The enamel was prepared by ED extraction, impregnation with acrylic plastic, decalcification and infiltration with gelatin.

Fig. 8.-The structure of the enamel rods and interprismatic substance can be observed in this thin section of plastic and gelatin embedded enamel. The embedding material has apparently replaced the organic and inorganic substances of enamel accurately. Observe the longitudinal and cross-sectioned enamel rods $(R)$ and the interprismatic substance $(I)$. 
The canals can be observed more elearly. In this plane, perpendicular to the long axis of the bone, the canals appear to form a regular network throughout the bone model. When a sample of cortical bone is sectioned along its longitudinal axis, rows of parallel haversian canals appear and numerous Folkmann's canals join them at nearly right angles (Fig. 4). Since the inorganic portion of this specimen is transparent, it is possible to visualize the channels deep in the bone, branching and joining the haversian canals of the surface plane. The channels are thus observed to be spaced fairly regularly and are completely interconnected. It was also of interest to study the relationship of the vascular channels to the haversian systems. Thus, one series of anorganic bone samples with vascular channels outlined with dye was decalcified and reimpregnated with plastic to approximately two thirds the depth of the block. This resulted in clearing of the specimen to the level of infiltration of the secondary impregnation. The remainder of the block contained the plastic skeleton of only the initial infiltration. Thus, such microscopic struetures as lamellae, lacunae, and canaliculi appeared. The relationship of colored plastic-filled blood vessels to haversian systems is seen in Figs. 5 and 6 . Volkmann's canals can be observed communicating with the haversian canals and with canals within interstitial lamellae. A haversian system with its central canal joined by two Volkmann's canals can be observed at high magnification in Fig. 6. This is an enlargement of the central area of Fig. 5. The plastic structure reveals the lacunae and canaliculi of the haversian system.

When it is desirable to study caleified tissues at even higher magnifications, plastic and gelatin-embedded specimens can be sectioned on the freezing microtome, mounted and studied. Five-micra sections of a plastic-gelatin model of enamel can be seen in Figs. 7 and 8 . This sample of enamel was from a mature erupted tooth and so consists mainly of inorganic salts which are represented by gelatin. The interprismatic substance between rods is believed to contain proportionately less inorganic salts and thus appears to be represented mainly by plastic which was infiltrated into the enamel following its treatment with ethylenediamine. The rod boundaries and the spaces occupied by interprismatic substance are more distinct than can be observed on thicker calcified ground sections.

\section{SUMMARY}

Organic material was extracted from samples of bone and teeth with ethylenediamine. The anorganic bone was impregnated with plastic containing a particulate dyestuff, decalcified, and impregnated with elear plastic to reveal a three-dimensional model of the vascular pattern. By only partial secondary plastic infiltration, the microstructure of bone can be studied in relation to this vaseular skeleton. By substituting a low molecular weight plastic and gelatin for the organic, water, and inorganic portions of dentin and enamel, it was found that this model could be sectioned on a microtome and an accurate histologic reproduction obtained. Application of these technies should result in further elucidation of the structure of calcified tissues. 
The authors wish to express their sincere appreciation to Miss Elizabeth Haley for sectioning and mounting of the three-dimensional replicas of bones and teeth.

\section{REFERENCES}

1. Williams, J. B., and Irvine, J. W., Jr.: Preparation of the Inorganic Matrix of Bone, Soience 119: 771, 1954 .

2. Peckham, S. C., and Losee, F. L.: Ethylenediamine vs. KOH-Glyeol in the Removal of the Organic Matter of Dentin, Research Report, Project NM 008 015.12.01, U. S. Naval Dental School, 1955.

3. Losee, F. L.: Microlamellae in Enamel Demonstrated by the Use of Ethylenediamine, D. Radiog. \& Photog. 29: 23, 1956.

4. Losee, F. L.: Personal communication, 1956. 